PROCEEDINGS OF THE

AMERICAN MATHEMATICAL SOCIETY

Volume 131, Number 11, Pages 3447-3456

S 0002-9939(03)06968-5

Article electronically published on February 20, 2003

\title{
THE SPECTRUM OF SCHRÖDINGER OPERATORS WITH POSITIVE POTENTIALS IN RIEMANNIAN MANIFOLDS
}

\author{
ZHONGWEI SHEN \\ (Communicated by Andreas Seeger)
}

\begin{abstract}
Let $M$ be a noncompact complete Riemannian manifold. We consider the Schrödinger operator $-\Delta+V$ acting on $L^{2}(M)$, where $V$ is a nonnegative, locally integrable function on $M$. We obtain some simple conditions which imply that inf $\operatorname{Spec}(-\Delta+V)$, the bottom of the spectrum of $-\Delta+V$, is strictly positive. We also establish upper and lower bounds for the counting function $N(\lambda)$.
\end{abstract}

\section{INTRODUCTION}

Let $M$ be a noncompact complete Riemannian manifold of dimension $d$. Let $V$ be a nonnegative, locally integrable function on $M$. Consider the quadratic form

$$
Q[\psi, \psi]=\int_{M}|\nabla \psi|^{2} d x+\int_{M} V|\psi|^{2} d x
$$

on $L^{2}(M)$, where $\nabla$ denotes the Riemannian gradient. Note that $\operatorname{Domain}(Q)=$ $\left\{\psi \in H^{1}(M): V^{1 / 2} \psi \in L^{2}(M)\right\}$. Clearly $Q[$,$] is a semibounded, symmetric closed$ form. It follows that there exists a unique self-adjoint operator, which we shall call $-\Delta+V$, such that

$$
Q[\phi, \psi]=\langle(-\Delta+V) \phi, \psi\rangle_{L^{2}(M)}
$$

for any $\phi \in \operatorname{Domain}(-\Delta+V)$ and $\psi \in \operatorname{Domain}(Q)$. Moreover, $C_{0}^{\infty}(M)$ is dense in $\operatorname{Domain}(Q)$.

In this paper we investigate certain spectral properties of $-\Delta+V$. In particular we obtain some simple conditions which imply that the bottom of spectrum

$$
E=\inf \operatorname{Spec}(-\Delta+V)
$$

is strictly positive. We also establish upper and lower bounds for the counting function $N(\lambda)$, the dimension of the spectral projection of $-\Delta+V$ on interval $[0, \lambda)$.

For a general Riemannian manifold, the question of positivity of $E$ was recently studied by Ouhabaz $[\mathrm{Ou}]$ under the condition $V \in L^{\infty}(M)$. Let $B(x, r)$ denote the

Received by the editors May 27, 2002.

2000 Mathematics Subject Classification. Primary 35P20, 35J10.

(C)2003 American Mathematical Society 
open ball in $M$ centered at $x$ with radius $r$. As in $\mathrm{Ou}$, we shall assume that there exist $r_{0}>0$ and $c_{1}, c_{2}>0$ such that

(a) The following $L^{2}$-Poincaré inequality holds on $M$ :

$$
\int_{B(x, r)}\left|u-\bar{u}_{B(x, r)}\right|^{2} d y \leq c_{1} r^{2} \int_{B(x, r)}|\nabla u|^{2} d y, \text { for all } x \in M \text { and } r \leq r_{0}
$$

where $u \in C^{\infty}(M)$ and

$$
\bar{u}_{B(x, r)}=\frac{1}{|B(x, r)|} \int_{B(x, r)} u d y .
$$

(b) $M$ satisfies the local doubling condition

$$
|B(x, 2 r)| \leq c_{2}|B(x, r)| \quad \text { for all } r \leq r_{0} \text { and } x \in M \text {. }
$$

It is known that if Ricci curvature of $M$ is bounded from below, then $M$ satisfies conditions (1.4) and (1.6) for all $r_{0}>0$ (with $c_{1}, c_{2}$ depending on $r_{0}$ ) [Bu, [C-G-T].

Theorem A. Let $M$ be a noncompact complete Riemannian manifold. Suppose that there exists $r_{0}>0$ such that conditions (1.4) and (1.6) hold with constants $c_{1}$, $c_{2}$. Also assume that there exist constants $c_{3}, c_{4}$ and $p \in(1, \infty]$ such that

$$
\inf _{x \in M} \frac{1}{\left|B\left(x, r_{0}\right)\right|} \int_{B\left(x, r_{0}\right)} V(y) d y=c_{3}>0
$$

and

$$
\left(\frac{1}{\left|B\left(x, r_{0}\right)\right|} \int_{B\left(x, r_{0}\right)}|V(y)|^{p} d y\right)^{1 / p} \leq \frac{c_{4}}{\left|B\left(x, r_{0}\right)\right|} \int_{B\left(x, r_{0}\right)} V(y) d y
$$

for all $x \in M$. Then

$$
E \geq \frac{c_{3}}{\max \left(2,2^{\frac{1}{p-1}}\right)\left(2 c_{1} c_{3} c_{4}^{\frac{p}{p-1}} r_{0}^{2}+1\right) c_{2}^{2}} .
$$

Remark 1.10. Under the conditions (1.4), (1.6), (1.7) and $V \in L^{\infty}(M)$, it is proved in $\mathrm{Ou}$ ] that $E>0$. Clearly, if $V \in L^{\infty}(M)$ and satisfies (1.7), then $V$ satisfies (1.8) with $p=\infty$ and $c_{4}=\|V\|_{\infty} / c_{3}$. Thus Theorem A contains Theorem 1 in $\mathrm{Ou}$. The main interest of our Theorem A lies in the much weaker assumption (1.8). We point out that in the Euclidean case it is possible to deduce (1.9) from the two-sided estimates in [Ma, Theorem 12.5.1, p. 465]. See also A-B for the special case $V \in L^{\infty}\left(\mathbb{R}^{d}\right)$.

Remark 1.11. The proof of Theorem A, which is different from that in $\mathrm{Ou}$, is fairly elementary. It is based on an idea of C. Fefferman-Phong [Fe. Main Lemma] for Schrödinger operators with nonnegative polynomial potentials in $\mathbb{R}^{d}$.

For $\lambda>0$, let $N(\lambda)$ denote the dimension of the spectral projection of $-\Delta+V$ on interval $[0, \lambda)$. In this paper we also establish upper and lower bounds for $N(\lambda)$ under additional conditions on potential $V$. To state the result, we first introduce the basic length scale

$$
\ell(x)=\inf \left\{r>0: \frac{r^{2}}{|B(x, r)|} \int_{B(x, r)} V(y) d y \geq r_{0}^{2} c_{3}\right\}
$$


for $x \in M$, where $c_{3}$ is the same constant as in (1.7). Clearly $0<\ell(x) \leq r_{0}$. The effective potential $v(x)$ is then defined by

$$
v(x)=\frac{1}{[\ell(x)]^{2}} .
$$

The definition of $v$ is similar to that of $\{m(x, V)\}^{2}$ in Sh1.

In the place of (1.8), we will assume that there exist $p \in(1, \infty]$ and $c_{5}, c_{6}>0$ such that

$$
\left\{\frac{1}{|B|} \int_{B}|V(y)|^{p} d y\right\}^{1 / p} \leq c_{5}\left\{\frac{1}{|B|} \int_{B} V(y) d y+\frac{1}{r^{2}}\right\}
$$

for all $B=B(x, r)$ with $0<r \leq r_{0}$ and

$$
\frac{r^{2}}{|B(x, r)|} \int_{B(x, r)} V(y) d y \leq c_{6} \eta\left(\frac{r}{R}\right)\left\{\frac{R^{2}}{|B(x, R)|} \int_{B(x, R)} V(y) d y+1\right\}
$$

for all $0<r<R \leq r_{0}$, where $\eta$ is a positive function on $[0,1]$ such that $\eta(t) \rightarrow 0$ as $t \rightarrow 0$.

Theorem B. Let $M$ be a noncompact complete Riemannian manifold. Suppose that there exists $r_{0}>0$ such that conditions (1.4) and (1.6) hold for all $r \leq r_{0}$ with constants $c_{1}$ and $c_{2}$. Let $V$ be a nonnegative function on $M$ satisfying (1.7). Further assume that $V$ satisfies conditions (1.14) and (1.15) for all $x \in M, 0<r<R \leq r_{0}$ and some $c_{5}, c_{6}>0, p>1$. Then there exist constants $C>0$ and $c>0$ depending only on $c_{i}, i=1,2,3,5,6$, and $p, \eta$ such that

$$
\begin{aligned}
& N(\lambda) \leq C \int_{\{x \in M: v(x) \leq C \lambda\}} \frac{d x}{\left|B\left(x, \frac{1}{\sqrt{\lambda}}\right)\right|}, \\
& N(\lambda) \geq c \int_{\{x \in M: v(x) \leq c \lambda\}} \frac{d x}{\left|B\left(x, \frac{1}{\sqrt{\lambda}}\right)\right|}
\end{aligned}
$$

for $\lambda$ sufficiently large.

If $M=\mathbb{R}^{d}$ and $p>d / 2$, then condition (1.14) implies condition (1.15) with $\eta(t)=t^{2-(d / p)}$ by Hölder inequality. In particular, if $V$ is in the reverse Hölder class, i.e.,

$$
\left(\frac{1}{|B|} \int_{B}|V|^{p} d x\right)^{1 / p} \leq \frac{C}{|B|} \int_{B} V d x \quad \text { for every ball } B \text { in } \mathbb{R}^{d}
$$

with exponent $p>d / 2(d \geq 2)$, then $V$ satisfies conditions (1.14) and (1.15). This is the case studied by the author in Sh2, Sh3 where estimates (1.16)-(1.17) are established. See also [Sh4. We should point out that [Sh3], Sh4] deal with the magnetic Schrödinger operator $(-i \nabla-\mathbf{a})^{2}+V$ in $\mathbb{R}^{d}$. The results in [Sh2], Sh3], Sh4] extend some earlier work on nonclassical eigenvalue asymptotics. We refer the reader to [Sh3] for references. We remark that in many cases it is possible to read off the leading order of $N(\lambda)$ as $\lambda \rightarrow \infty$ from estimates like (1.16)-(1.17). This is especially useful in the case of degenerate potentials when the classical CwickelLieb-Rosenbljum bound, which may be extended to manifolds satisfying the global Sobolev inequality Li-Ya, Le-So, fails to yield any information. In particular it follows that under the assumptions in Theorem B, the Schrödinger operator $-\Delta+V$ has a discrete spectrum if and only if for some $x_{0} \in M, v(x) \rightarrow \infty$ as $\operatorname{dist}\left(x, x_{0}\right) \rightarrow \infty$. See Corollary 3.14 . 
Finally, we note that conditions (1.14)-(1.15), which allow $V$ to vanish on certain open sets, are much weaker than the reverse Hölder condition (1.18). Theorem B is new even in the Euclidean case.

Throughout this paper we will assume that there exists $r_{0}>0$ such that the $L^{2}$-Poincaré inequality (1.4) and the doubling condition (1.6) hold for all $x \in M$ and $0<r \leq r_{0}$ with constants $c_{1}, c_{2}$.

\section{Proof of Theorem A}

For $x \in M$ and $0<r \leq r_{0}$, we define

$$
V(x, r)=\frac{1}{2|B(x, r)|} \int_{B(x, r)} \frac{V(y)}{2 c_{1} r^{2} V(y)+1} d y .
$$

The proof of Theorem A is based on the following lemma which is a direct extension of the Main Lemma in [Fe. See also [Sh4, Theorem 3.1].

Lemma 2.2. Let $B=B\left(x_{0}, r\right)$ where $x_{0} \in M$ and $0<r \leq r_{0}$. Then, for any $u \in C^{\infty}(B)$,

$$
V\left(x_{0}, r\right) \int_{B}|u|^{2} d x \leq \int_{B}|\nabla u|^{2} d x+\int_{B} V|u|^{2} d x .
$$

Proof. The proof is similar to that in the Euclidean case [Fe].

Using expansion and the Poincaré inequality (1.4), we obtain

$$
\frac{1}{2|B|} \iint_{B \times B}|u(x)-u(y)|^{2} d x d y=\int_{B}\left|u-\bar{u}_{B}\right|^{2} d x \leq c_{1} r^{2} \int_{B}|\nabla u|^{2} d x .
$$

This, together with

$$
\frac{1}{|B|} \iint_{B \times B} V(y)|u(y)|^{2} d x d y=\int_{B} V(x)|u(x)|^{2} d x
$$

gives

$$
\begin{aligned}
& \frac{1}{|B|} \iint_{B \times B}\left\{\frac{1}{2 c_{1} r^{2}}|u(x)-u(y)|^{2}+V(y)|u(y)|^{2}\right\} d x d y \\
& \leq \int_{B}|\nabla u|^{2} d x+\int_{B} V|u|^{2} d x .
\end{aligned}
$$

It follows that

$$
\begin{gathered}
\frac{1}{2|B|} \int_{B} \min \left(\frac{1}{2 c_{1} r^{2}}, V(y)\right) d y \cdot \int_{B}|u(x)|^{2} d x \\
\leq \int_{B}|\nabla u|^{2} d x+\int_{B} V|u|^{2} d x
\end{gathered}
$$

Since $\min (a, b) \geq a b /(a+b)$, we obtain

$$
\frac{1}{2|B|} \int_{B} \min \left(\frac{1}{2 c_{1} r^{2}}, V(y)\right) d y \geq V\left(x_{0}, r\right) .
$$

The proof is complete.

Lemma 2.3. Suppose that $V$ satisfies (1.7)-(1.8) for some $p>1$ with constants $c_{3}, c_{4}$. Then

$$
\inf _{x \in M} V\left(x, r_{0}\right) \geq \frac{c_{3}}{\max \left(2,2^{\frac{1}{p-1}}\right)\left(2 c_{1} c_{3} c_{4}^{\frac{p}{p-1}} r_{0}^{2}+1\right)}
$$


Proof. Let $q=p /(p-1)$ and $B=B\left(x, r_{0}\right)$. By Hölder's inequality,

$$
\begin{aligned}
\int_{B} V(y) d y & =\int_{B}\left(\frac{V(y)}{2 c_{1} r_{0}^{2} V(y)+1}\right)^{1 / q}\left(2 c_{1} r_{0}^{2} V(y)+1\right)^{1 / q}|V(y)|^{1 / p} d y \\
& \leq\left\{\int_{B} \frac{V(y) d y}{2 c_{1} r_{0}^{2} V(y)+1}\right\}^{1 / q}\left\{\int_{B}\left(2 c_{1} r_{0}^{2} V(y)+1\right)^{p / q} V(y) d y\right\}^{1 / p}
\end{aligned}
$$

If $p \geq 2$, then $p / q=p-1 \geq 1$. By Minkowski's inequality,

$$
\begin{aligned}
& \left\{\int_{B}\left(2 c_{1} r_{0}^{2} V(y)+1\right)^{p / q} V(y) d y\right\}^{q / p} \\
& \leq 2 c_{1} r_{0}^{2}\left\{\int_{B}|V(y)|^{p} d y\right\}^{q / p}+\left\{\int_{B} V(y) d y\right\}^{q / p} .
\end{aligned}
$$

It follows that

$$
\begin{aligned}
V\left(x, r_{0}\right) & =\frac{1}{2|B|} \int_{B} \frac{V(y) d y}{2 c_{1} r_{0}^{2} V(y)+1} \\
& \geq \frac{\left(\frac{1}{|B|} \int_{B} V(y) d y\right)^{q}}{2\left\{\frac{1}{|B|} \int_{B}\left(2 c_{1} r_{0}^{2} V(y)+1\right)^{\frac{p}{q}} V(y) d y\right\}^{q / p}} \\
& \geq \frac{\left(\frac{1}{|B|} \int_{B} V(y) d y\right)^{q}}{4 c_{1} r_{0}^{2}\left(\frac{1}{|B|} \int_{B}|V(y)|^{p} d y\right)^{q / p}+2\left(\frac{1}{|B|} \int_{B} V(y) d y\right)^{q / p}} \\
& \geq \frac{\left(\frac{1}{|B|} \int_{B} V(y) d y\right)^{q}}{4 c_{1} r_{0}^{2} c_{4}^{q}\left(\frac{1}{|B|} \int_{B} V(y) d y\right)^{q}+2\left(\frac{1}{|B|} \int_{B} V(y) d y\right)^{q / p}} \\
& =\frac{1}{4 c_{1} r_{0}^{2} c_{4}^{q}+2\left(\frac{1}{|B|} \int_{B} V(y) d y\right)^{-1}} \\
& \geq \frac{c_{3}}{4 c_{1} c_{3} c_{4}^{q} r_{0}^{2}+2} .
\end{aligned}
$$

If $1<p<2$, then $p / q=p-1<1$. In place of Minkowski's inequality, we use

$$
\begin{aligned}
& \left\{\int_{B}\left(2 c_{1} r_{0}^{2} V(y)+1\right)^{\frac{p}{q}} V(y) d y\right\}^{q / p} \\
& \quad \leq 2^{\frac{q}{p}-1}\left\{2 c_{1} r_{0}^{2}\left(\int_{B}|V(y)|^{p} d y\right)^{q / p}+\left(\int_{B} V(y) d y\right)^{q / p}\right\} .
\end{aligned}
$$

The rest is the same as in the case $p \geq 2$. The proof is finished.

We are now in a position to give the

Proof of Theorem A. Let $\alpha$ denote the constant in right-hand side of (2.4). By Lemma 2.2, we have

$$
\alpha \int_{B\left(x_{0}, r_{0}\right)}|\psi|^{2} d x \leq \int_{B\left(x_{0}, r_{0}\right)}|\nabla \psi|^{2} d x+\int_{B\left(x_{0}, r_{0}\right)} V|\psi|^{2} d x
$$


for any $\psi \in C_{0}^{\infty}(M)$. We divide both sides of $(2.5)$ by $\left|B\left(x_{0}, r_{0}\right)\right|$ and then integrate the resulting inequality with respect to $x_{0}$ over $M$. By Fubini's Theorem, we obtain

$$
\alpha \int_{M}|\psi(x)|^{2} h(x) d x \leq \int_{M}|\nabla \psi(x)|^{2} h(x) d x+\int_{M} V(x)|\psi(x)|^{2} h(x) d x
$$

where

$$
h(x)=\int_{B\left(x, r_{0}\right)} \frac{d x_{0}}{\left|B\left(x_{0}, r_{0}\right)\right|} .
$$

Note that, if $x_{0} \in B\left(x, r_{0}\right)$, then $B\left(x_{0}, r_{0}\right) \subset B\left(x, 2 r_{0}\right)$ and $B\left(x, r_{0}\right) \subset B\left(x_{0}, 2 r_{0}\right)$. By the doubling condition (1.6), this implies that

$$
\begin{aligned}
& h(x) \geq \int_{B\left(x, r_{0}\right)} \frac{d x_{0}}{\left|B\left(x, 2 r_{0}\right)\right|} \geq \frac{1}{c_{2}} \\
& h(x) \leq c_{2} \int_{B\left(x, r_{0}\right)} \frac{d x_{0}}{\left|B\left(x_{0}, 2 r_{0}\right)\right|} \leq c_{2} \int_{B\left(x, r_{0}\right)} \frac{d x_{0}}{\left|B\left(x, r_{0}\right)\right|}=c_{2} .
\end{aligned}
$$

It follows that

$$
\frac{\alpha}{c_{2}^{2}} \int_{M}|\psi|^{2} d x \leq \int_{M}|\nabla \psi|^{2} d x+\int_{M} V|\psi|^{2} d x
$$

for any $\psi \in C_{0}^{\infty}(M)$. By the minimax principle, $E=\inf \operatorname{Spec}(-\Delta+V) \geq \alpha / c_{2}^{2}$. This completes the proof.

Remark 2.7. For a manifold $M$ with bounded geometry, Kondrat'ev and Shubin K-S gave a necessary and sufficient condition on $V$ for the spectrum of $-\Delta+V$ to be discrete. This extends a theorem of A. M. Molchanov for the case $M=\mathbb{R}^{d}$ Ma Theorem 12.5 .4 , p. 466]. The necessary and sufficient condition involves the Newtonian capacity in geodesic coordinates. We point out that if $M$ is of bounded geometry, one may deduce Theorem A from Proposition 5.3 in $[\mathrm{K}-\mathrm{S}$ ] on the lower bound for the first Neumann eigenvalue of $-\Delta+V$ on a ball.

\section{Proof of Theorem B}

Let $\lambda>0$. Suppose $1 / \sqrt{\lambda} \leq r_{0}$. Since $M$ satisfies the doubling condition (1.6), there exist a sequence $\left\{x_{j}\right\}_{j \in \mathbb{N}}$ of points in $M$ and a positive number $K$ depending on $c_{2}$ such that for $B_{j}=B\left(x_{j}, \frac{1}{\sqrt{\lambda}}\right)$, we have $M=\bigcup_{j} B_{j}, \frac{1}{2} B_{j} \cap \frac{1}{2} B_{k}=\emptyset$ if $j \neq k$, and each $x \in M$ is contained in at most $K$ balls $B_{j}$. We refer the reader to $\mathrm{Ou}$. for a proof of above statement.

Let $\Lambda$ denote the set of all $j \in \mathbb{N}$ such that

$$
\frac{1}{\left|B_{j}\right|} \int_{B_{j}} V d x \leq \lambda
$$

We begin with a lower bound for $N(\lambda)$.

Lemma 3.2. There exists $C>0$ depending only on $c_{2}$ such that

$$
N(C \lambda) \geq \text { the number of elements in } \Lambda
$$

for all $\lambda \geq 1 / r_{0}^{2}$.

Proof. For each $j \in \Lambda$, let

$$
\psi_{j}(x)= \begin{cases}\frac{1}{2 \sqrt{\lambda}}-\operatorname{dist}\left(x, x_{j}\right), & x \in \frac{1}{2} B_{j}, \\ 0, & \text { otherwise. }\end{cases}
$$


Then $\psi_{j} \in \operatorname{Domain}(Q)$. It is easy to see that

$$
Q\left[\psi_{j}, \psi_{j}\right] \leq C\left|B_{j}\right| \leq C \lambda\left\|\psi_{j}\right\|_{L^{2}(M)}^{2} .
$$

Since $\left\{\frac{1}{2} B_{j}\right\}_{j \in \Lambda}$ are mutually disjoint, $\left\{\psi_{j}\right\}_{j \in \Lambda}$ is an orthogonal set in $L^{2}(M)$. This, together with (3.3), implies that $N(C \lambda) \geq$ the number of elements in $\Lambda$ by the minimax principle.

Lemma 3.4. Suppose that $V$ satisfies condition (1.15) for all $0<r<R \leq r_{0}$. Also assume that $V$ satisfies (1.7). Then there exist $C>0, c>0$ depending only on $c_{2}, c_{3}, c_{6}, r_{0}$ and $\eta$ such that

$$
N(C \lambda) \geq c \int_{\{x \in M: v(x) \leq c \lambda\}} \frac{d x}{\left|B\left(x, \frac{1}{\sqrt{\lambda}}\right)\right|}
$$

for $\lambda$ sufficiently large.

Proof. By the doubling condition (1.6),

$$
\int_{B_{j}} \frac{d x}{\left|B\left(x, \frac{1}{\sqrt{\lambda}}\right)\right|} \approx 1 .
$$

It follows from Lemma 3.2 that

$$
\begin{aligned}
N(C \lambda) & \geq \text { the number of elements in } \Lambda \\
& \geq c \sum_{j \in \Lambda} \int_{B_{j}} \frac{d x}{\left|B\left(x, \frac{1}{\sqrt{\lambda}}\right)\right|} \\
& \geq c \int_{\cup_{j} B_{j}} \frac{d x}{\left|B\left(x, \frac{1}{\sqrt{\lambda}}\right)\right|} .
\end{aligned}
$$

To finish the proof, it suffices to show that

$$
\{x \in M: v(x) \leq c \lambda\} \subset \bigcup_{j \in \Lambda} B_{j}
$$

for some $c>0$. To this end, we note that by (1.15), if $x \in B_{j}$ for some $j \notin \Lambda$ and $R=\frac{2}{\delta \sqrt{\lambda}}$, then

$$
\begin{aligned}
& \frac{R^{2}}{|B(x, R)|} \int_{B(x, R)} V(y) d y \geq \frac{1}{c_{5} \eta(\delta)} \frac{\left(\frac{2}{\sqrt{\lambda}}\right)^{2}}{\left|B\left(x, \frac{2}{\sqrt{\lambda}}\right)\right|} \int_{B\left(x, \frac{2}{\sqrt{\lambda}}\right)} V(y) d y-1 \\
& \quad \geq \frac{c}{\eta(\delta)}-1>r_{0}^{2} c_{3}
\end{aligned}
$$

if $\delta$ is small. It follows from the definition that $\ell(x)<\frac{2}{\delta \sqrt{\lambda}}$. Thus $v(x)>c \lambda$ for all $x \in \bigcup_{j \notin \Lambda} B_{j}$. This implies that

$$
\{x \in M: v(x) \leq c \lambda\} \subset M \backslash \bigcup_{j \notin \Lambda} B_{j} \subset \bigcup_{j \in \Lambda} B_{j} .
$$

The proof is complete.

Next we estimate $N(\lambda)$ from above. 
Lemma 3.7. Suppose that $V$ satisfies (1.14). Then there exists $c>0$ depending only on $c_{1}, c_{2}, c_{5}$ and $p$ such that

$$
N(c \lambda) \leq \text { the number of elements in } \Lambda
$$

for all $\lambda \geq 1 / r_{0}^{2}$.

Proof. Let $r=1 / \sqrt{\lambda} \leq r_{0}$. Note that by (1.14), if $j \notin \Lambda$,

$$
c \lambda \leq\left(\frac{1}{\left|B_{j}\right|} \int_{B_{j}}|V|^{p} d x\right)^{1 / p} \leq \frac{C}{\left|B_{j}\right|} \int_{B_{j}} V d x .
$$

It then follows from the proof of Lemma 2.3 that, if $j \notin \Lambda$,

$$
\begin{aligned}
V\left(x_{j}, r\right) & \geq \frac{c\left(\frac{1}{\left|B_{j}\right|} \int_{B_{j}} V d x\right)^{q}}{\frac{1}{\lambda}\left(\frac{1}{\left|B_{j}\right|} \int_{B_{j}}|V|^{p} d x\right)^{q / p}+\left(\frac{1}{\left|B_{j}\right|} \int_{B_{j}} V d x\right)^{q / p}} \\
& \geq \frac{c\left(\frac{1}{\left|B_{j}\right|} \int_{B_{j}} V d x\right)^{q}}{\frac{1}{\lambda}\left(\frac{1}{\left|B_{j}\right|} \int_{B_{j}} V d x\right)^{q}+\left(\frac{1}{\left|B_{j}\right|} \int_{B_{j}} V d x\right)^{q / p}} \\
& =\frac{c\left(\frac{1}{\left|B_{j}\right|} \int_{B_{j}} V d x\right)}{\frac{1}{\lambda}\left(\frac{1}{\left|B_{j}\right|} \int_{B_{j}} V d x\right)+1} \\
& \geq c \lambda .
\end{aligned}
$$

By Lemma 2.2, this implies that

$$
c \lambda \int_{B_{j}}|\psi|^{2} d x \leq \int_{B_{j}}|\nabla \psi|^{2} d x+\int_{B_{j}} V|\psi|^{2} d x
$$

for all $j \notin \Lambda$ and $\psi \in C_{0}^{\infty}(M)$.

For $j \in \Lambda$, we use Poincaré inequality (1.4) to obtain

$$
c \lambda \int_{B_{j}}|\psi|^{2} d x \leq \int_{B_{j}}|\nabla \psi|^{2} d x+\int_{B_{j}} V|\psi|^{2} d x
$$

if $\psi \perp \chi_{B_{j}}$. Combining (3.9) and (3.10), we see that if $\psi \in C_{0}^{\infty}(M)$ and $\psi \perp \chi_{B_{j}}$ for all $j \in \Lambda$, then

$$
c \lambda \int_{M}|\psi|^{2} d x \leq \int_{M}|\nabla \psi|^{2} d x+\int_{M} V|\psi|^{2} d x
$$

where we have also used the fact that $1 \leq \sum_{j} \chi_{B_{j}} \leq K$. Lemma 3.7 now follows from (3.11) by the minimax principle.

Lemma 3.12. Suppose that $V$ satisfies the conditions (1.7), (1.14) and (1.15). Then there exist $C>0$ and $c>0$ depending on $c_{1}, c_{2}, c_{3}, c_{5}, c_{6}, \eta$ and $p$ such that

$$
N(c \lambda) \leq C \int_{\{x \in M: v(x) \leq C \lambda\}} \frac{d x}{\left|B\left(x, \frac{1}{\sqrt{\lambda}}\right)\right|}
$$

for $\lambda$ sufficiently large. 
Proof. By Lemma 3.7,

$$
\begin{aligned}
N(c \lambda) & \leq \text { the number of elements in } \Lambda \\
& \leq C \sum_{j \in \Lambda} \int_{\frac{1}{2} B_{j}} \frac{d x}{\left|B\left(x, \frac{1}{\sqrt{\lambda}}\right)\right|} \\
& \leq C \int_{\bigcup_{j \in \Lambda} \frac{1}{2} B_{j}} \frac{d x}{\left|B\left(x, \frac{1}{\sqrt{\lambda}}\right)\right|} .
\end{aligned}
$$

Thus it suffices to show that

$$
\bigcup_{j \in \Lambda} \frac{1}{2} B_{j} \subset\{x \in M: v(x) \leq C \lambda\}
$$

for some $C>0$. To see (3.13), we use condition (1.14). Note that if $x \in \frac{1}{2} B_{j}$ for some $j \in \Lambda$, then $B\left(x, \frac{1}{2 \sqrt{\lambda}}\right) \subset B_{j}$ and

$$
\begin{aligned}
\frac{\left(\frac{\delta}{2 \sqrt{\lambda}}\right)^{2}}{\left|B\left(x, \frac{\delta}{2 \sqrt{\lambda}}\right)\right|} & \int_{B\left(x, \frac{\delta}{2 \sqrt{\lambda}}\right)} V(y) d y \\
& \leq c_{6} \eta(\delta)\left\{\frac{\left(\frac{1}{2 \sqrt{\lambda}}\right)^{2}}{\left|B\left(x, \frac{1}{2 \sqrt{\lambda}}\right)\right|} \int_{B\left(x, \frac{1}{2 \sqrt{\lambda}}\right)} V(y) d y+1\right\} \\
& \leq C \eta(\delta)<c_{3} r_{0}^{2}
\end{aligned}
$$

if $\delta \in\left(0, \delta_{0}\right)$ and $\delta_{0}$ is small. It follows from the definition that $\ell(x) \geq \delta_{0} /(2 \sqrt{\lambda})$. Thus $v(x) \leq C \lambda$ for all $x \in \bigcup_{j \in \Lambda} \frac{1}{2} B_{j}$. (3.13) is then proved and the proof of Lemma 3.12 is complete.

Proof of Theorem B. By the doubling condition (1.6), it is easy to see that the upper bound (1.16) follows from Lemma 3.12, while the lower bound (1.17) follows from Lemma 3.4.

Corollary 3.14. Under the same assumptions as in Theorem $\mathrm{B}$, the spectrum of $-\Delta+V$ is discrete if and only if for some $x_{0} \in M, v(x) \rightarrow \infty$ as $\operatorname{dist}\left(x, x_{0}\right) \rightarrow \infty$.

Proof. Suppose that for some $x_{0} \in M, v(x) \rightarrow \infty$ as $\operatorname{dist}\left(x, x_{0}\right) \rightarrow \infty$. For any $\lambda$ sufficiently large, there exists $R_{\lambda}>0$ such that $\{x \in M: v(x)<C \lambda\} \subset B\left(x_{0}, R_{\lambda}\right)$. It follows from (1.16) that

$$
N(\lambda) \leq C\left|B\left(x_{0}, R_{\lambda}\right)\right| \sup _{x \in B\left(x_{0}, R_{\lambda}\right)} \frac{1}{\left|B\left(x, \frac{1}{\sqrt{\lambda}}\right)\right|}<\infty .
$$

Thus $N(\lambda)<\infty$ for any $\lambda>0$. It follows that the spectrum of $-\Delta+V$ is discrete.

Next suppose that for some $x_{0} \in M, v(x) \nrightarrow \infty$ as $\operatorname{dist}\left(x, x_{0}\right) \rightarrow \infty$. Then there exist $\varepsilon>0$ and a sequence $\left\{x_{j}\right\}$ of points in $M$ such that $v\left(x_{j}\right)<1 / \varepsilon^{2}$ and $\operatorname{dist}\left(x_{j}, x_{0}\right) \rightarrow \infty$ as $j \rightarrow \infty$. It follows that $\ell\left(x_{j}\right)>\varepsilon$. Consequently, by definition,

$$
\frac{1}{\left|B\left(x_{j}, \varepsilon\right)\right|} \int_{B\left(x_{j}, \varepsilon\right)} V(y) d y<\frac{c_{3} r_{0}^{2}}{\varepsilon^{2}} \text {. }
$$

By the proof of Lemma 3.2, there exists $\psi_{j} \in \operatorname{Domain}(Q)$ such that $\left\|\psi_{j}\right\|_{L^{2}(M)}=1$, $\operatorname{supp} \psi_{j} \subset B\left(x_{j}, \varepsilon\right)$ and $Q\left[\psi_{j}, \psi_{j}\right] \leq C / \varepsilon^{2}$. Since $\operatorname{dist}\left(x_{j}, x_{0}\right) \rightarrow \infty$ as $j \rightarrow \infty$, by the minimax principle, we have $N\left(C / \varepsilon^{2}\right)=\infty$. This completes the proof. 


\section{REFERENCES}

[A-B] W. Arendt and C.J.K. Batty, Exponential stability of a diffusion equation with absorption, Diff. Integral Eqs. 6 (1993), 1009-1024. MR 94k:35038

[Bu] P. Buser, A note on the isoperimetric constant, Ann. Sci. École Norm. Sup. 15 (1982), 213-230. MR 84e:58076

[C-G-T] J. Cheeger, M. Gromov, and M. Taylor, Finite propagation speed, kernel estimates for functions of the Laplace operator, and the geometry of complete Riemannian manifolds, J. Diff. Geom. 17 (1982), 15-53. MR 84b:58109

[Da] E. B. Davies, Heat Kernels and Spectral Theory, Cambridge Univ. Press, 1990. MR 92a:35035

[Fe] C. Fefferman, The uncertainty principle, Bull. Amer. Math. Soc. 9 (1983), 129-206. MR 85f:35001

[K-S] V. Kondratev and M. Shubin, Discreteness of spectrum for the Schrödinger operators on manifolds of bounded geometry, Operator Theory: Advances and Applications 110 (1999), 185-226. MR 2001c:58030

[Le-So] D. Levin and M. Solomyak, Rozenblum-Lieb-Cwikel inequality for Markov generators, J. d'Analyse Math. 71 (1997), 173-193. MR 98j:47090

[Li-Ya] P. Li and S-T. Yau, On the Schrödinger equation and the eigenvalue problem, Comm. Math. Phys. 88 (1983), 309-318. MR 84k:58225

[Ma] V. G. Maz'ya, Sobolev Spaces, Springer Verlag, Berlin, 1985. MR 87g:47056

$[\mathrm{Ou}] \quad$ E. M. Ouhabaz, The spectral bound and principal eigenvalues of Schrödinger operators on Riemannian manifolds, Duke Math. J. 110(1) (2001). MR 2002i:58038

[Re-Si] M. Reed and B. Simon, Methods of Modern Mathematical Physics, vol. II, Academic Press, 1975. MR 58:12429b

[Sh1] Z. Shen, $L^{p}$ estimates for Schrödinger operators with certain potentials, Ann. Inst. Fourier 45(2) (1995), 513-546. MR 96h:35037

[Sh2] - On the eigenvalue asymptotics of Schrödinger operators, unpublished (1995).

[Sh3] Eigenvalue asymptotics and exponential decay of eigenfunctions for Schrödinger operators with magnetic fields, Trans. Amer. Math. Soc. 348(11) (1996), 4465-4488. MR 97d:35162

[Sh4] $\quad$ On bounds of $N(\lambda)$ for a magnetic Schrödinger operator, Duke Math. J. 94(3) (1998), 479-507. MR 99e:35171

Department of Mathematics, University of Kentucky, Lexington, Kentucky 40506

E-mail address: shenz@ms.uky.edu 